\title{
Magnet-only loudspeaker magnetic circuits: a solution for significantly lower current distortion
}

\author{
Benoit Merit ${ }^{1}$, Antonin Novak ${ }^{2 *}$ \\ ${ }^{1}$ Orkidia Audio, 64310 Ascain, France \\ ${ }^{2}$ Laboratoire d'Acoustique de I'Université du Mans, LAUM - UMR 6613 CNRS, Le Mans Université, Avenue Olivier \\ Messiaen, 72085 LE MANS CEDEX 9, France \\ *antonin.novak@univ-lemans.fr \\ *https://ant-novak.com
}

\begin{abstract}
This paper presents a study case which leads to a significant decrease of current distortion caused by the magnetic circuit of the loudspeaker: the solution consists in removing all of the nonlinear soft-iron pieces that are used in traditional loudspeaker motors. Thus, in the loudspeaker motors we study, the static magnetic field is only created thanks to a combination of permanent magnets. This combination leads to the realization of the so-called magnet-only loudspeakers. Two loudspeakers are compared in the study: the first sample is a mass-produced 6.5 inch loudspeaker. The second sample is built replacing the magnetic circuit of the first loudspeaker by the magnet-only structure. Thus, the moving part, including the voice-coil, the diaphragm and the suspensions, is kept identical for both samples, leading to a comparison of both loudspeakers only based on their motor. The loudspeaker with the magnet-only motor shows significantly lower current distortion than the original loudspeaker. A possible explanation of such a decrease of distortion is given, based on results of a set of measurements with blocked voice-coil.
\end{abstract}

The archived file is not the final published version of the article B. Merit \& A. Novak (2015), "Magnet-Only Loudspeaker Magnetic Circuits: A Solution for Significantly Lower Current Distortion", Journal of the Audio Engineering Society. Vol. 63(6), pp. 463-474.

The definitive publisher-authenticated version is available online at $\mathrm{https}: / / \mathrm{doi} . \mathrm{org} / 10.17743 / \mathrm{jaes} .2015 .0051$, Readers must contact the publisher for reprint or permission to use the material in any form. 


\section{INTRODUCTION}

The basic structure of electrodynamic loudspeaker motors has remained unchanged for almost 100 years. Most are still made of a permanent magnet or electromagnet and iron pole pieces (top plate and T-yoke) to canalize the magnetic flux $B$ into the air-gap where the voice-coil moves. The basic principle of the electro-mechanical conversion realized by this loudspeaker motor when fed with the voltage signal $u(t)$ can be described using the following linear equation $[1,2,3,4,5]$

$$
u(t)=R e \cdot i(t)+L e \frac{d i(t)}{d t}+B l \cdot \frac{d x(t)}{d t},
$$

where $R e$ is the voice-coil resistance, $L e$ is the voice-coil inductance and $B l$ is the force factor, if $l$ is the lenght of the voice-coil immersed in the magnetic flux $B$. The interaction between the electrical current $i(t)$ running through the voice-coil and the magnetic flux $B$ creates a force $F(t)=B l i(t)$ that causes the displacement $x(t)$ of the voice-coil.

However, a real loudspeaker motor is far from the ideal one described by the linear equations. Due to the inherent behavior of a classical electromagnetic motor, electrical current running through the voice-coil can be distorted, yielding audible degradation of the output acoustical signal generated by the loudspeaker. Regarding the motor part, this distortion is usually supposed to be caused only by variations of the force factor $B l$ and the inductance $L e$ due to both the current running through the voice-coil and the displacement of this latter [6].

There exists many solutions to decrease the distortion caused by the loudspeaker motor. Some solutions are based on current feedback $[7,8]$, linearizing the current. Others modify directly the structure of the motor in such a way that they limit the eddy currents in the pole pieces ; a solution based on a fixed copper cylinder in the air gap, patented in [9], has shown its ability to decrease the current distortion by a factor of 10 [10]. A different structure based on iron-nickel alloys rings has shown a similar decrease of distortion [11]. The use of shorting rings is now the most common way of controlling the voice-coil inductance to reduce the nonlinearities of a loudspeaker $[12,13,14,15]$. A slightly different solution using steel pole pieces tipped with a polymer-based magnetic-powder composite lead to a 10 to $15 \mathrm{~dB}$ attenuation of the third-harmonic [16].

A few years ago, a new concept of magnet-only loudspeaker, also called "ironless loudspeaker", has been proposed to decrease the non-linearities of the loudspeaker motors. Such loudspeakers are called magnet-only because their magnetic circuit is totally made of rare-earth permanent magnets, such as $N d F e B$ magnets. The elimination of iron from transducer motors is first presented in patent [17] and next can be found in [18, 19, 20,21]. Advantages of such ironless structures have been shortly highlighted in [22] and [23]. More recently, Remy [24] presented loudspeaker distortions reduction through the use of bonded magnet motors.

It has already been shown in $[23,25]$ that magnet-only magnetic circuits can lead to constant force factors whatever the displacements of the voice-coil, improving the linearity of the transducer. But, at high frequencies, corresponding to small displacements, and for low levels of supplying current, the often put forward displacementand current-dependencies of the force factor and of the inductance fail to explain the observed decrease of distortion. In this paper, we try to associate the decrease of current distortion with a decrease of variation of electrical parameters measured when the voice-coil is mechanically blocked (blocked parameters).

Thus, the aim of this paper is twofold. First, it shows that a magnet-only loudspeaker can significantly improve the motor linearity. Second, it clarifies how much a decrease of current distortion can be associated with a decrease of the variations of the loudspeaker motors blocked parameters. With this aim in view, the usually put forward qualitative advantages of magnet-only motors are pointed out. Then, we introduce the concept of apparent resistance Re and apparent inductance Le and we present a set of measurements drawing the combined frequency-dependencies of apparent blocked parameters with displacement-dependence and with current level-dependence.

The measurements are applied for a comparison between two versions of a mass-produced loudspeaker. The first version is made of a classical magnetic circuit with soft-iron pieces. The second version is the same loudspeaker 
whose magnetic circuit has been replaced by a magnet-only one, inspired from structures presented in [26]. Thus, contrary to preceding works on magnet-only loudspeakers [20] - [26], such an experiment allows us to keep the moving part including the voice-coil and the suspensions identical for both loudspeakers leading to a fair comparison of both motors.

\section{WHY USE MAGNET-ONLY MOTORS : THEORETICAL BACKGROUND}

\subsection{Dependence of inductance on current intensity}

The inductance of a voice-coil in a conventional iron-using loudspeaker motor depends nonlinearly on the current running through the voice-coil. This represents a source of distortion in loudspeakers [6]. In a linear approximation, the inductance of a coil is considered to be proportional to the mean permeability $\mu$ of the material the coil magnetic flux flows through $[28,29]$. The permeability of a material is given directly by the slope of its BH-curve, which draws the level of magnetic induction $B$ in the material as a function of magnetic excitation $H$. When a time-varying magnetic excitation $H(t)$ is imposed to a material, the time varying permeability $\mu(t)$ must be considered; it is defined then by the local slope of the BH-curve at time $t$.

In a loudspeaker, the magnetic flux of the voice-coil can flow through air, iron pieces and permanent magnet. If a time-varying current $i(t)$ runs through the voice-coil, it creates a time-varying magnetic excitation $H(t)$, that is added to the static magnetic excitation imposed by the permanent magnet.

- The BH-curve of air is a straight line whose slope $\mu_{0}=4 \pi \cdot 10^{-7} H \cdot m^{-1}$ is the permeability of air, independent of the magnetic excitation $H_{a}(t)$. No matter how much magnetic excitation varies in air, its permeability remains the same.

- Similarly, the BH-curve of actual rare-earth $N d F e B$ magnets is a very large hysteresis loop whose dynamic relation between magnetic induction $B_{m}(t)$ and magnetic excitation $H_{m}(t)$ remains linear for a very large region of $H$ [27]:

$$
B_{m}(t)=J+\mu_{m} H_{m}(t),
$$

where $J$ is the residual induction and $\mu_{m} \approx \mu_{0}$ is the permeability of the rare-earth permanent magnets.

- BH-curve of iron is highly hysteretic, and the working point imposed by the magnet is mostly close to the saturation of iron ; around this working point, the local variations of BH-curve imposed by the voice-coil are also hysteretic [11]. This is why the mean permeability of iron varies with the current running through the voice-coil [6].

Since classical loudspeaker motors uses iron parts which instantaneous permeability varies with the current running through the voice-coil, the inductance of the voice-coil varies with the current intensity as well. On the other hand, since the magnet-only motors are made of hard magnets and air, for which the permeability remains the same whatever the level of current running through the voice-coil, the inductance of a voice-coil placed in a magnet-only motor should not depend anymore on the supplying current intensity.

\subsection{Dependence of inductance on voice-coil displacement}

The inductance of a voice-coil from a classical motor is known to depend on the coil axial position and consequently on its displacement. When the voice-coil moves back and forth in a classical motor with iron, the proportion of the dynamic magnetic field created by the voice-coil and passing through iron, air and magnet changes as well. The magnetic permeability of iron is highly different to the one of air and permanent magnets and thus the mean permeability varies according to the voice-coil position [6]. 
This dependence also creates an additional motive force, called reluctance force $[21,30]$. By considering that properties of the voice-coil do not vary with the supplying current intensity, the total motive force is often written as the sum of the Laplace force $F_{L}=B l i$ and the reluctance force $F_{R}=\frac{1}{2} \frac{d L e(z)}{d z} i^{2}$ [30], with the axis $O z$ standing for the axis of revolution of the loudspeaker. The nonlinear force $F_{R}$ is proportional to the inductance gradient and to the square of the current intensity, and tends to set the voice-coil in its position of maximal inductance [27]. It is then well understood that this force is a potential source of consequent distortions at high level of current, no matter the displacement of the voice-coil.

In contrast, the magnetic circuit of a magnet-only loudspeaker consists only of rare-earth magnets and air, whose permeability $\mu_{m}$ and $\mu_{0}$ are almost equal. Thus, the mean permeability does not change whatever the position of the voice-coil and consequently the inductance of such a voice-coil should not vary either.

\subsection{Frequency dependence and Eddy Currents}

On the one hand, the top plate and the T-yoke of classical loudspeakers are made of iron whose electrical conductivity is high. Thus, when the time-varying magnetic field of the voice-coil flows through these pieces, eddy currents are created and run through them to oppose the magnetic field of the voice-coil. Their effects in loudspeakers are well known [31] and even exploited to control the voice-coil impedance $[32,13]$. They are the origin of a variation of both the inductance and the resistance of the voice coil with frequency of the input signal. Scott [31] and later Vanderkooy [33] showed that the inductance of a voice-coil becomes complex in presence of eddy currents: the imaginary part of this complex inductance is responsible for the drop of the voice-coil apparent resistance with frequency, responsible for heating increase, while the real part contributes to the variation of its apparent inductance with frequency.

On the other hand, the conductivity of the rare-earth $N d F e B$ magnets used in magnet-only loudspeaker is very low. This should lead to almost no eddy currents and thus to both resistance and inductance almost independent on the frequency [27].

\section{DEVICE UNDER TEST}

In order to show how the magnet-only motor may improve the linearity of the loudspeaker, we provide a set of measurement results on two versions of a loudspeaker. The first version is a sample of a mass-produced 6.5 inch loudspeaker whose motor is made of a classical magnetic circuit with soft-iron pieces. The second version is the same loudspeaker whose motor is replaced by a magnet-only one.

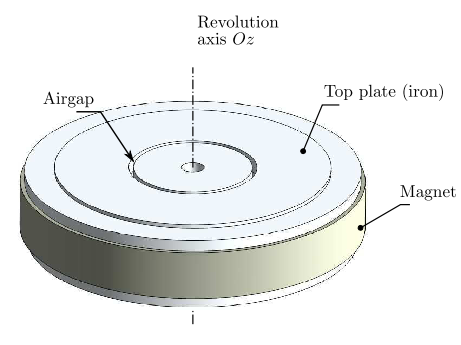

(a)

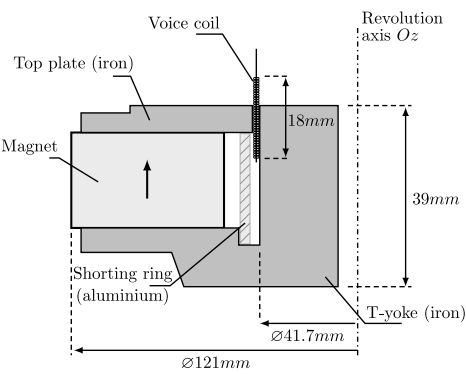

(b)

Figure 1. (a) Magnetic circuit of the original loudspeaker and (b) cross-section view of its magnetic circuit. The arrow in magnet indicates its magnetization direction. 


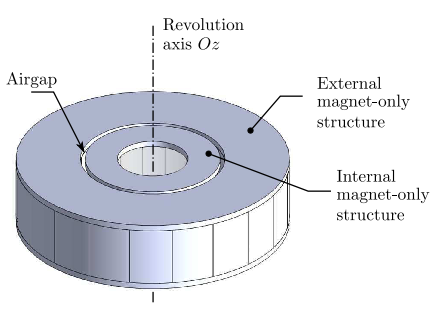

(a)

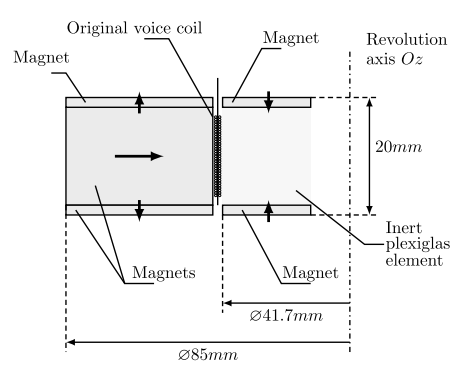

(b)

Figure 2. (a) Magnet-only magnetic circuit of the modified loudspeaker and (b) cross-section view of its magnetic circuit. The arrows in magnets indicate their magnetization direction.

\subsection{Motor description}

The original loudspeaker is equipped with a traditional production model magnetic circuit, made of ferrite magnet and iron, and using an aluminum shorting ring (Fig. 1). After measuring the original loudspeaker, the motor is replaced by a prototype of a magnet-only magnetic circuit, whose design is inspired from [26] (Fig. 2) ${ }^{1}$. Such an experiment allows us to keep the moving part, including the voice-coil and the suspensions, identical for both loudspeakers.

\subsection{Loudspeaker properties}

The magnet-only magnetic circuit has been designed in such a way that the force factor $\operatorname{Bl}(x)$ of the magnet-only loudspeaker is as close as possible to the original loudspeaker.

As depicted in Figs. 1 and Fig. 2, the original loudspeaker uses an overhung voice-coil, whilst the magnetonly motor, as described in [26], can only use an underhung voice-coil. Moreover, the design of the magnetonly circuit is limited by the distance between the voice-coil and the diaphragm. This is why the overhung and underhung configurations are sought to be equivalent for small displacements, but not functionally equivalent otherwise, resulting in different $B l(x)$ curves for large displacements.

To estimate the force factor $B l$, we measure first the radial component of the magnetic induction $B$ along the entire path of the voice-coil using a FW BELL, 5180 Gauss / Tesla Meter with a probe FW BELL 1X and we calculate the $B l$ as a function of the position $x$ as described in [34]. Both measured force factors are depicted in Fig. 3. The value of the force factor in the rest position equals $B l=10.4 \mathrm{~N} / \mathrm{A}$ for the original loudspeaker and $B l=10.5 \mathrm{~N} / \mathrm{A}$ for the modified loudspeaker. As depicted in Fig. 3, they are both almost constant within the displacement region from -1.5 to $1.5 \mathrm{~mm}$ in which all the measurements are performed.

On the other hand, Fig. 3 shows that the force factor $B l$ differs outside the -1.5 to $1.5 \mathrm{~mm}$ displacement range. That is why the voice-coil displacements should remain smaller than $\pm 1.5 \mathrm{~mm}$ for our study. This shall ascertain that the measured differences between both versions of the studied loudspeaker only stand in the electrical properties of the motor ( $R e$ and $L e$ ).

To verify that both versions are equivalent, we provide a traditional electrical impedance curves measured using a swept-sine signal and a $470 \mathrm{~m} \Omega$ Current Shunt (N4L HF003). Both, the amplitude and the phase of the impedance (Fig. 4) remains almost equal for both loudspeakers up to $1 \mathrm{kHz}$. At higher frequencies $(>1 \mathrm{kHz})$ however, we note a slightly different behavior of both the amplitude and the phase of the impedance. This difference denotes

\footnotetext{
${ }^{1}$ It can be seen in Fig. 2 that the magnet-only magnetic circuit includes a ring of plexiglas. This piece is only made to help maintaining the chosen magnet assembly. Plexiglas does not interfere the measurements because it is inert, i.e. its conductivity is almost null and its permeability is that of the air.
} 


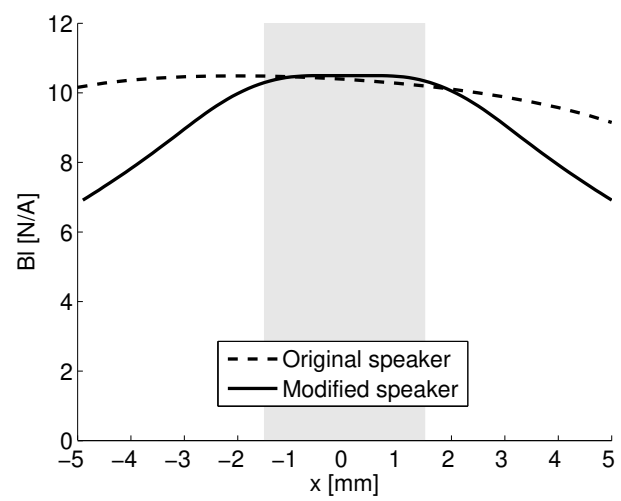

Figure 3. Measured force factor created by the traditional magnetic circuit (dashed line) and by the magnet-only magnetic circuit (thick line) as a function of position. The gray area from $-1.5 \mathrm{~mm}$ to $1.5 \mathrm{~mm}$ denotes the displacement range in which both force factors are almost identical and in which both loudspeakers can be fairly compared.

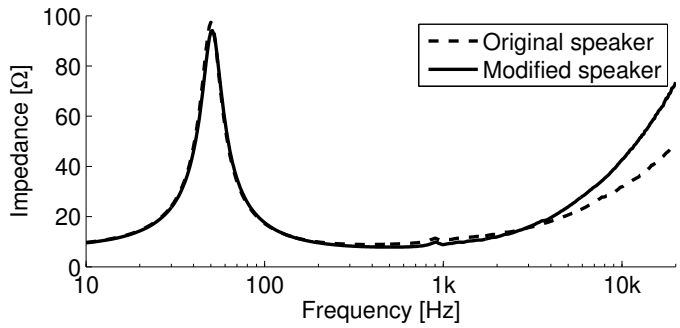

(a)

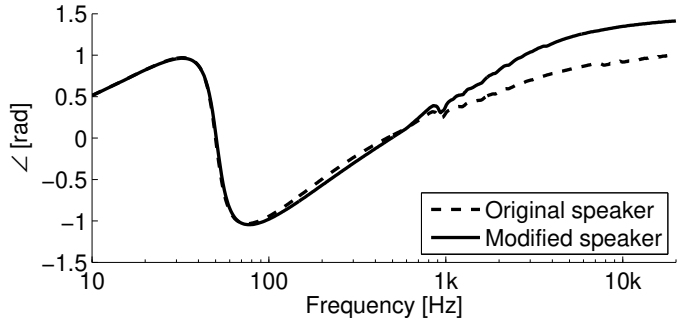

(b)

Figure 4. Impedance of both loudspeakers as a function of frequency; (a) absolute value and (b) phase. Measurements realized with a moving (non-blocked) voice coil.

an alteration of the inductive behavior, as explained in section 4.3.

Both versions being almost equivalent from the traditional linear point of view, we focus next on the nonlinearities of both motors and on the variation of block resistance $R e$ and blocked inductance $L e$ as a function of displacement, current and frequency. Both force factors $B l$ are equivalent for the $\pm 1.5 \mathrm{~mm}$ displacement range in which the following measurements are made. Moreover, both mechanical parts are identical for both versions of the studied loudspeaker. Then the possible differences in measurement results should be caused only by the electrical behavior of the motor, implying only resistance $R e$ and inductance $L e$. 


\section{EXPERIMENT DESCRIPTION AND RESULTS}

In this section, we present the results of the comparative study of both motors from the electrical properties point of view. First, we measure the harmonic distortion of the current and we compare it for both loudspeakers. Then, to understand the observed differences between both distortion measurements, the electrical impedance (apparent resistance $R e$ and apparent inductance $L e$ ) of the blocked voice-coil are measured as a function of position, current and frequency. Since the voice-coil is blocked, the equation (1) becomes

$$
u_{\text {blocked }}(t)=R e \cdot i(t)+L e \frac{d i(t)}{d t}
$$

The term apparent is used to suggest that both parameters $R e$ and Le can be measured directly using a simple impedance-meter. They are derived from the real and the imaginary part of the measured impedance.

\subsection{Tools of Instrumentation}

For the free voice-coil measurements presented in section 4.2 we use a Sound card RME Fireface 400 for generation and acquisition of signals. The Sound card has the Signal to Noise ratio (SNR) 110 dB RMS. A low-distortion power amplifier Devialet D-Premier with the Signal to Noise ratio (SNR) 130 dB RMS is used to amplify the output signal. The harmonic, as well as the inter-modulation distortion of the amplifier is less than $0.001 \%$. The amplifier and the sound card are connected using a digital S/PDIF interface. The current is measured using a $470 \mathrm{~m} \Omega$ Current Shunt (N4L HF003).

The measurement of resistance $R e$ and inductance $L e$ of the blocked-voice coil, presented in sections 4.3 and 4.4 is performed using a WAYNE KERR Inductance Analyzer 3255B with a measurement accuracy of $0.1 \%$.

The voice-coil is blocked mechanically using small inert plexiglas rings of different heights inserted on the bottom of the airgap below the former. The membrane is next held by a plastic clamp during the measurement in such a way that the former is blocked to prevent any motion. The position of the voice coil is verified using a vernier scale with a precision of $0.02 \mathrm{~mm}$

Finally, to measure the current distortion of the blocked-voice coil, presented in section 4.5, we use again the $470 \mathrm{~m} \Omega$ Current Shunt (N4L HF003).

\subsection{Harmonic distortion}

A classical way to measure the harmonic distortion is to excite the nonlinear system by a harmonic signal with a given amplitude and frequency $f_{0}$ and to analyze the frequency components $m f_{0}$ of the distorted output signal that are integer multiples $m$ of the fundamental frequency $f_{0}$. An example of such a measurement is shown in Fig. 5, where spectra of current responses are compared for both loudspeakers (original and modified one) being excited by a $1 \mathrm{kHz}$ sine-wave signal of amplitude $3 \mathrm{~V}$ ( $1 \mathrm{~W}$ excitation). Note that such a signal causes a coil displacement $\approx 10 \mu \mathrm{m}$ so that the possible differences in the measured distortion cannot be explained by any variation of force factor.

To compare the harmonic distortion of the current as a function of frequency, we use a swept-sine signal excitation $[35,36]$ and we observe separately the 2 nd and the 3 rd harmonic. Both samples are excited with a $3 \mathrm{~V}$ swept-sine signal from $150 \mathrm{~Hz}$ to $10 \mathrm{kHz}$, frequencies for which the displacement is much smaller than $1.5 \mathrm{~mm}$. The frequencydependence of relative level of second and third harmonics of current are depicted in Fig. 6 as a function of the excitation frequency. In Fig. 5 units $\mathrm{dBuA}$ (current in $\mathrm{dB}$ relative to $1 \mu \mathrm{A}$ ) are chosen on current axis, whilst in Fig. 6 the level is plotted as relative to the fundamental harmonic.

It is obvious from Figs. 5 and 6 that the distortion is much lower for magnet-only structure than for the original motor. The single-sine excitation (Fig. 5) reveals the reduction of the second and third harmonic by 18 and $41 \mathrm{~dB}$ respectively. The other higher harmonics are completely hidden in the background noise level $(0 \mathrm{dBuA})$ in the case of magnet-only motor as depicted in Fig. 5b. 


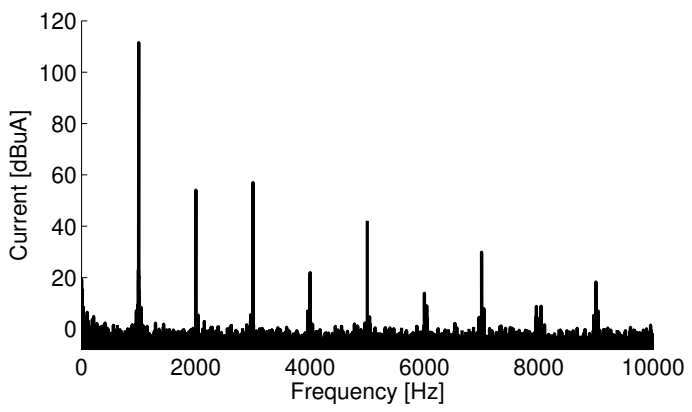

(a)

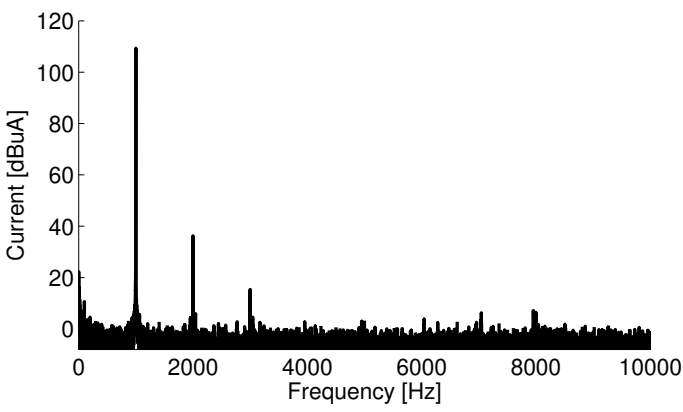

(b)

Figure 5. Comparison of spectra of current responses to $1 \mathrm{kHz}$ sine-wave excitation (at 1 Watt). a) original motor, b) magnet-only motor. Measurements realized with a moving (non-blocked) voice coil.

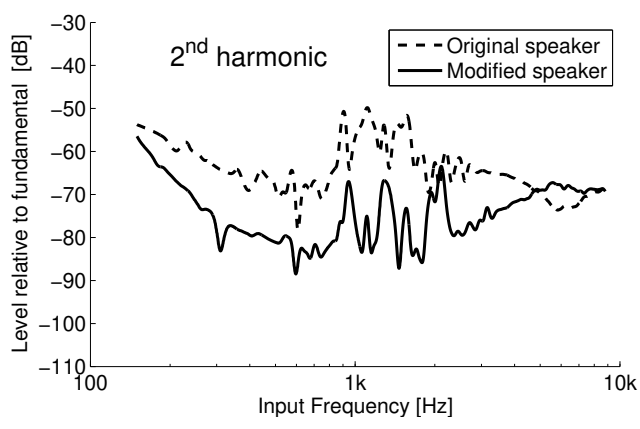

(a)

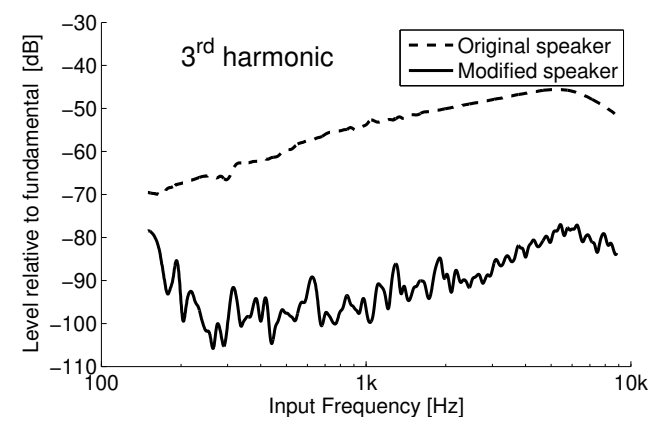

(b)

Figure 6. Relative harmonic distortion of the current as a function of frequency; (a) 2nd harmonic and (b) 3rd harmonic, when excited with a $3 \mathrm{~V}$ swept-sine. Measurements realized with a moving (non-blocked) voice coil.

The second and third harmonic analysis in a large frequency range (Fig. 6), reveals a higher reduction of distortion for mid and high frequencies. For frequencies higher than $150 \mathrm{~Hz}$, the second harmonic (Fig. 6a) is reduced on average by $10 \mathrm{~dB}$ and the third harmonic (Fig. 6b) by $40 \mathrm{~dB}$.

The results depicted in Figs. 5 and 6 show that replacing the original motor by the one with the magnet-only structure leads to an important reduction of current distortion. Since the moving part is unchanged for both loudspeakers the reduction of the distortion is most likely caused by the modification of the motor part of the loudspeaker (force factor $\mathrm{Bl}$, apparent resistance $R e$ and apparent inductance $L e$ ). Moreover, since the factor $\mathrm{Bl}$ is kept almost identical for both versions of the loudspeaker within the displacement range $\pm 1.5 \mathrm{~mm}$ for which the measurements are realized, the reduction of distortion is supposed to be linked only with the changes in apparent resistance $R e$ and apparent inductance $L e$.

Thus, we investigate in the following the variations of the electrical impedance (apparent resistance $R e$ and apparent inductance $L e$ ) of the blocked voice-coil with its position, current and frequency of both versions of the loudspeaker.

\subsection{Frequency-dependence of blocked parameters variations with position}

First, we pay attention to the variations of apparent resistance $R e$ and apparent inductance Le of the blocked voice-coil as a function of both, position and frequency. Intensity of the current flowing through the voice-coil is set to $20 \mathrm{~mA}$, low enough to avoid any current nonlinearities.

For each blocked position of the voice-coil in the air-gap, starting from $-5 \mathrm{~mm}$ to $+5 \mathrm{~mm}$ with $0.5 \mathrm{~mm}$ step, 


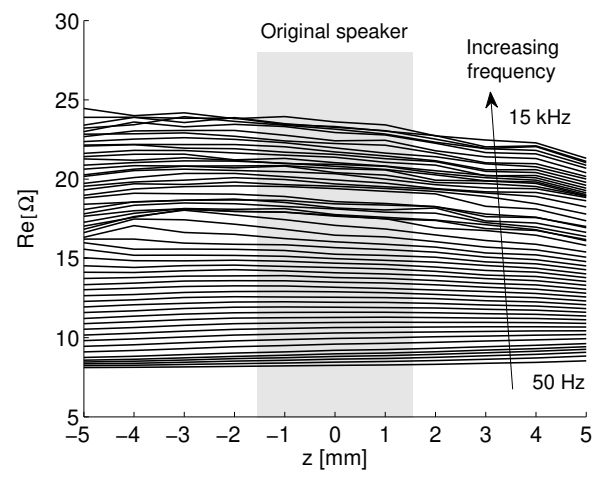

(a)

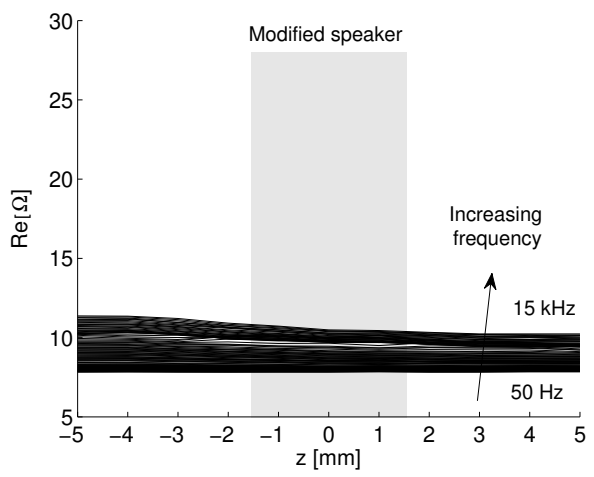

(b)

Figure 7. Apparent resistance Re as a function of voice-coil position for the original loudspeaker (a) and for the modified loudspeaker (b). Measurements realized with a non-moving (blocked) voice coil. The gray area from $-1.5 \mathrm{~mm}$ to $1.5 \mathrm{~mm}$ denotes the displacement range in which both force factors are almost identical and in which both loudspeakers can be fairly compared.

the blocked parameters are measured for several frequencies between $50 \mathrm{~Hz}$ and $15 \mathrm{kHz}$. For both speakers, the real part of the blocked impedance, i.e. the apparent resistance Re of the voice-coil, is presented in Fig. 7, and the imaginary part divided by angular frequency $\omega$, i.e. the apparent inductance $L e$ of the voice-coil, is presented in Fig. 8. In both figures, the gray area from $-1.5 \mathrm{~mm}$ to $1.5 \mathrm{~mm}$ denotes the displacement range in which both force factors are almost identical and in which both loudspeakers can be fairly compared.

In the case of the original motor, apparent resistance Re (Fig. 7a) and apparent inductance Le (Fig. 8a) exhibit variations with both, position of the blocked voice-coil and frequency. First, frequency-dependence of the blocked parameters is significant, whatever the position of the voice-coil in the magnetic circuit. When the voice-coil is set at $x=-1.5 \mathrm{~mm}$, the variation of the apparent resistance $R e$ goes from $8 \Omega$ at $50 \mathrm{~Hz}$ to $24 \Omega$ at $15 \mathrm{kHz}$ and the variation of the apparent inductance $L e$ goes from $1.25 \mathrm{mH}$ at $50 \mathrm{~Hz}$ to $0.35 \mathrm{mH}$ at $15 \mathrm{kHz}$. Besides, there is a clear position dependence of the apparent inductance $L e$.

When looking at the variations of blocked parameters of the modified speaker (Fig. $7 \mathrm{~b}$ and $8 \mathrm{~b}$ ), it is clear that both variations of apparent resistance and apparent inductance are reduced. Frequency-dependence of blocked parameters $R e$ and $L e$ becomes insignificant compared to the original loudspeaker, whatever the position of the voice-coil. When the voice coil is set at $x=-1.5 \mathrm{~mm}$, the maximal variation of the apparent resistance Re goes from $8 \Omega$ to $12 \Omega$ and the maximal variation of the apparent inductance $L e$ goes from $0.6 \mathrm{mH}$ to $0.7 \mathrm{mH}$. Besides, the dependence of the blocked resistance $R e$ and the blocked inductance $L e$ with the position $x$ is almost negligible.

\subsection{Frequency-dependence of blocked parameters variations with current}

In order to measure the variations of blocked parameters with current level, the voice-coil is blocked in its rest position $(x=0)$, and its apparent blocked parameters are measured for several frequencies and current levels. Four different frequencies are chosen: $50 \mathrm{~Hz}, 500 \mathrm{~Hz}, 5 \mathrm{kHz}$ and $15 \mathrm{kHz}$. The current intensity is increased from $20 \mathrm{~mA}$ to $0.5 \mathrm{~A}$ with a $20 \mathrm{~mA}$ step (thick lines in Figs. 9 and 10), and then decreased back from $0.5 \mathrm{~A}$ to $20 \mathrm{~mA}$ with the same step (thin lines) to ensure the results are not biased by the Joule heating. If the apparent resistance would increase due to heating of the voice-coil with increasing current, the results for a small level of current would differ when measured when the voice-coil is "cold" and when measured after the voice coil has been heated by a higher level of current. Nevertheless, as one can see in Fig. 9, the variation of apparent resistance due to the Joule heating is almost negligible. 


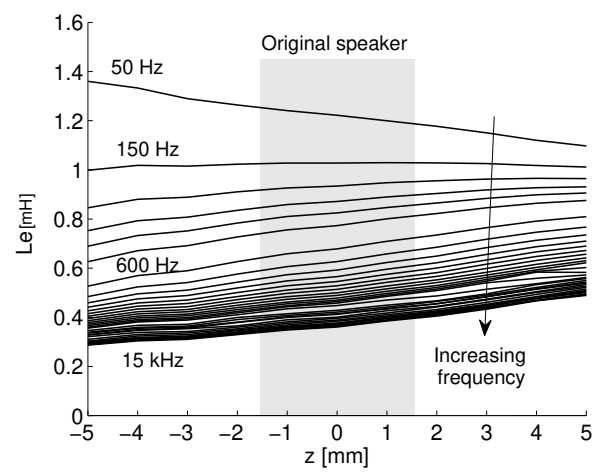

(a)

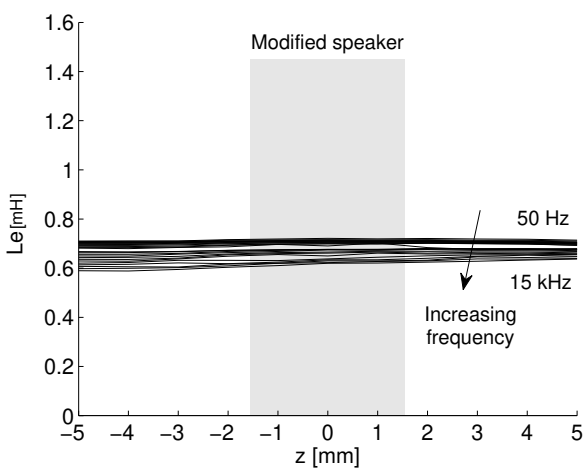

(b)

Figure 8. Apparent inductance $L e$ as a function of voice-coil position for the original loudspeaker (a) and for the modified loudspeaker (b). Measurements realized with a non-moving (blocked) voice coil. The gray area from $-1.5 \mathrm{~mm}$ to $1.5 \mathrm{~mm}$ denotes the displacement range in which both force factors are almost identical and in which both loudspeakers can be fairly compared.

The variations of the blocked parameters are depicted in Figs. 9 and 10 relatively to the values measured for the lowest value of the current level $(20 \mathrm{~mA})$. In the case of the original loudspeaker, both the apparent resistance and the apparent inductance vary with current, the variation being more important at higher frequencies, where the blocked resistance increases by $10 \%$ and the blocked inductance increases by $20 \%$.

In the case of the modified loudspeaker, both the apparent resistance and the apparent inductance are almost constant compared to the original loudspeaker case, whatever the current level. The variation of both resistance and inductance with current does not exceed $0.2 \%$ for all measured frequencies.

\subsection{Harmonic distortion as a function of excitation level}

In the last experiment, the voice-coil is kept blocked in its rest position and both original and modified loudspeakers are supplied with increasing voltage from $1 \mathrm{~V}$ to $30 \mathrm{~V}$ (equivalent to $100 \mathrm{~W}$ of input power) at $1 \mathrm{kHz}$. The harmonic distortion of the current is measured, and the level of five higher harmonics relative to the fundamental are depicted as a function of the driving voltage in Fig. 11.

The current distortion of the modified loudspeaker is almost negligible compared to the current distortion of the original one. As expected, the distortion of the original loudspeaker increases with the driving level; the 3rd harmonic, already significant at low input levels, approaches $-40 \mathrm{~dB}$ relative to the fundamental. On the other hand, the higher harmonics are kept under $-75 \mathrm{~dB}$ relative to the fundamental in the case of the modified loudspeaker even for a high power input levels $(100 \mathrm{~W})$.

\section{Discussion}

The results presented above have shown a decrease of the nonlinear distortion of the loudspeaker whose motor has been replaced by a prototype of a magnet-only magnetic circuit. Since the moving part of the loudspeaker, including the voice coil, remains unchanged for both studied cases, the reduction of the distortion is considered to be caused by the magnet-only motor. Moreover, since the magnet-only motor has been designed in such a way that the force factor $B l(x)$ is almost the same for both loudspeakers within the measurement displacement range, the reduction of the distortion seems to be linked to the different apparent resistance Re and inductance Le only.

In Figs. 7 and 8 the original blocked resistance and inductance have shown a huge dependence on frequency, whilst this dependence almost disappeared in the case of the magnet-only loudspeaker. These results show that 


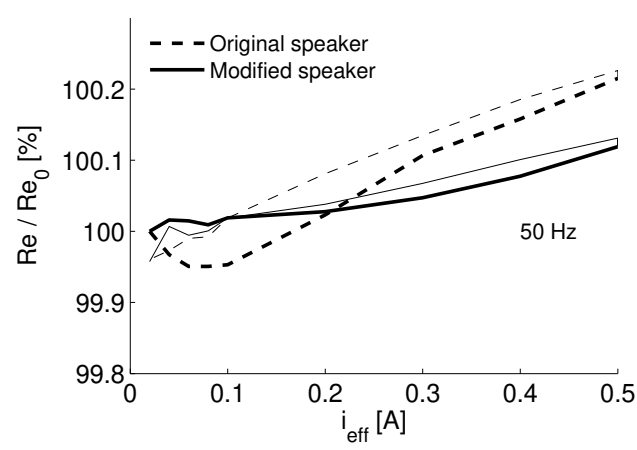

(a)

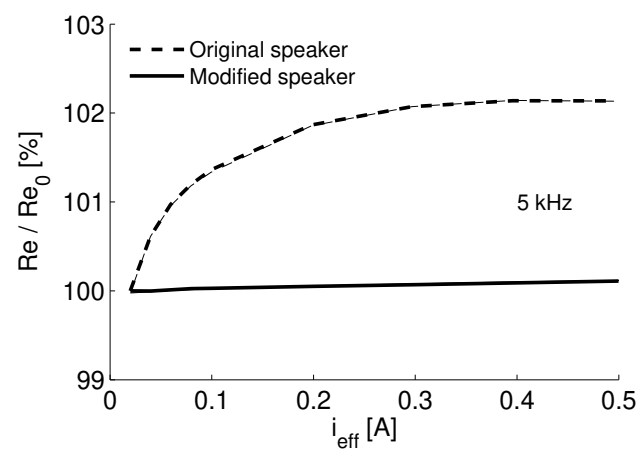

(c)

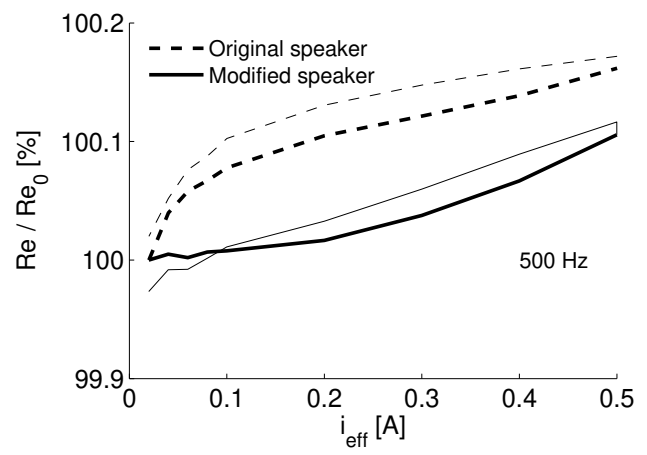

(b)

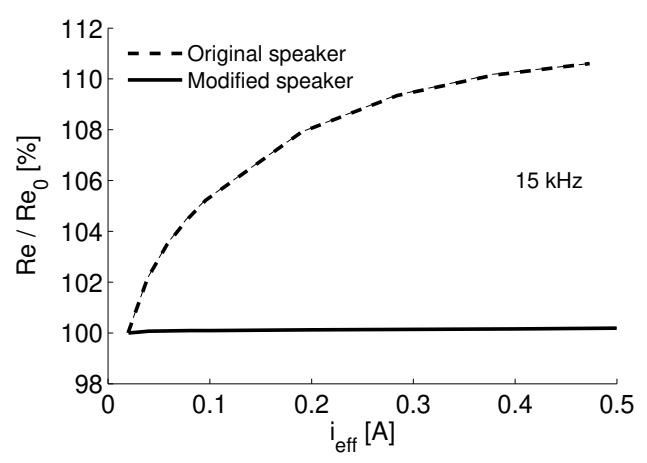

(d)

Figure 9. Apparent resistance Re of the blocked voice-coil of both original (dashed line) and modified (solid line) loudspeakers, as a function of increasing current (thick line) or decreasing current (thin line), for frequencies: $50 \mathrm{~Hz}(\mathrm{a}), 500 \mathrm{~Hz}$ (b), $5 \mathrm{kHz}$ (c) and $15 \mathrm{kHz}$ (d). Results are represented in [\%] relatively to the apparent resistance $R_{e 0}$ measured for the lowest value of the current level $(20 \mathrm{~mA})$. Measurements realized with a non-moving (blocked) voice coil.

characterizing the position-dependence of inductance, the influence of the frequency should be taken into account. More especially, looking at the results obtained with the original loudspeaker, it is important to note from Fig. 8a that the slope of the variation of $L e$ with $x$ coordinate depends also on the frequency of the input signal. The slope of the curve obtained at $50 \mathrm{~Hz}$ is negative while it is positive for frequencies higher than $150 \mathrm{~Hz}$. That means that, in usual working conditions of this loudspeaker, the reluctance force $F_{R}=\frac{1}{2} \frac{d L e(z)}{d z} i^{2}$ [30] can pull the voice-coil inside the magnetic circuit at low frequencies while it can push it out of the magnetic circuit at high frequencies, potentially being a source of a nonlinear distortion.

The variations of the apparent resistance $R e$ and the apparent inductance $L e$ with frequency and with position of the voice-coil in the air-gap for the original loudspeaker may be explained by two different phenomena: 


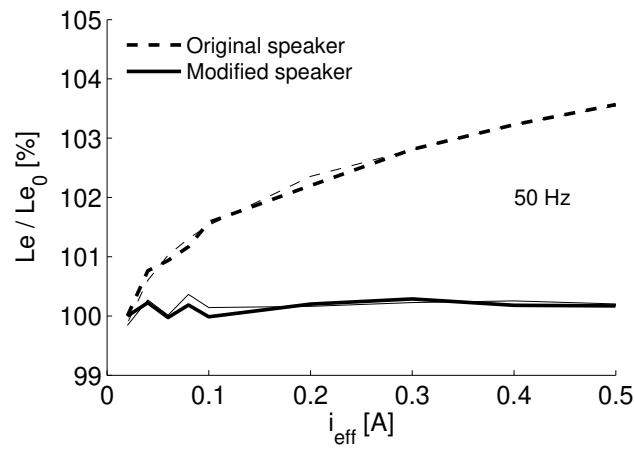

(a)

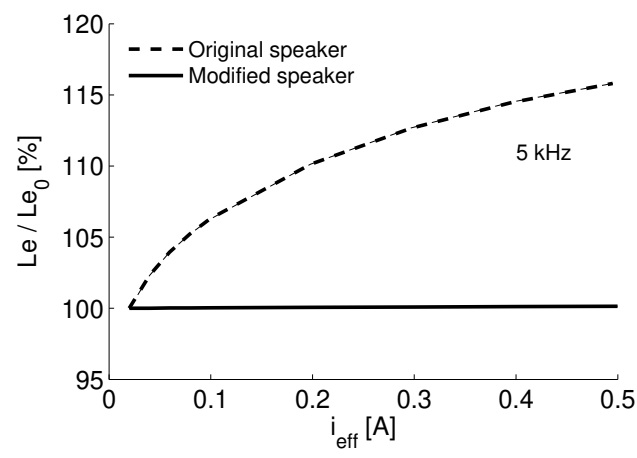

(c)

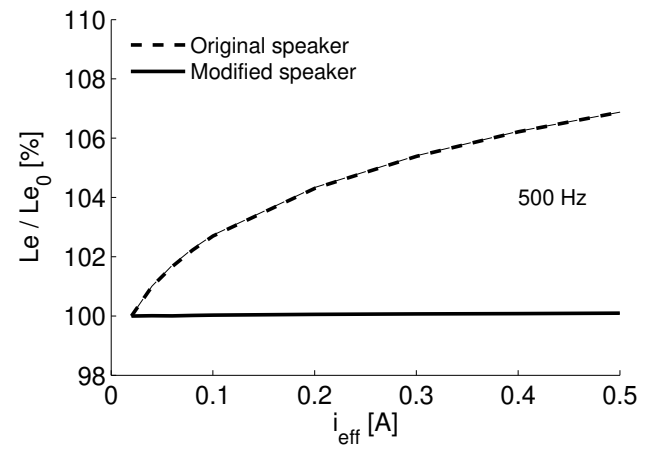

(b)

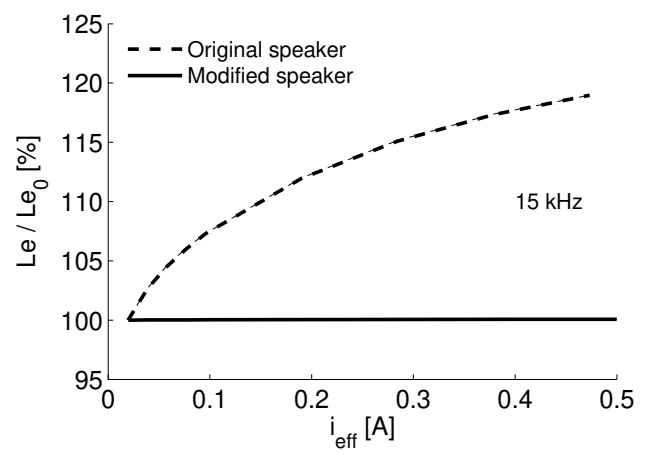

(d)

Figure 10. Apparent inductance Le of the blocked voice-coil of both original (dashed line) and modified (solid line) loudspeakers, as a function of increasing current (thick line) or decreasing current (thin line), for frequencies: $50 \mathrm{~Hz}(\mathrm{a}), 500 \mathrm{~Hz}$ (b), $5 \mathrm{kHz}$ (c) and $15 \mathrm{kHz}$ (d). Results are represented in [\%] relatively to the apparent inductance $L_{e 0}$ measured for the lowest value of the current level $(20 \mathrm{~mA})$. Measurements realized with a non-moving (blocked) voice coil. 


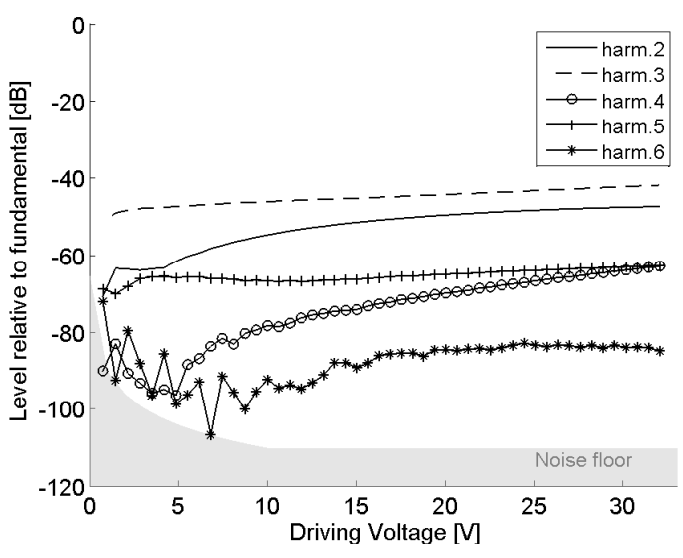

(a)

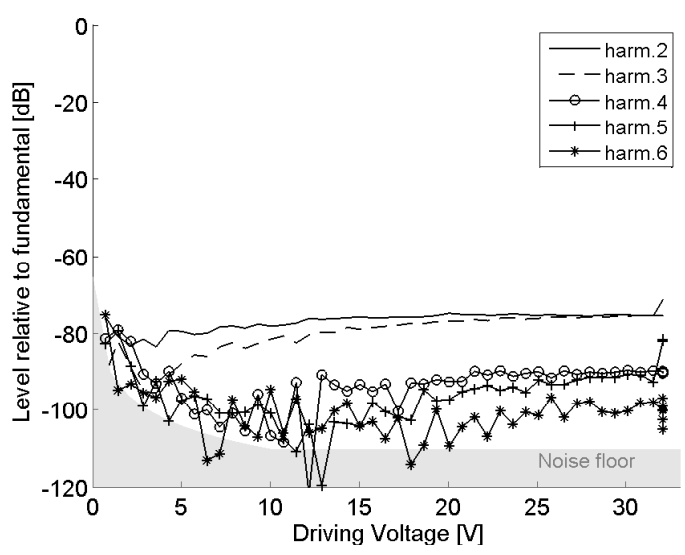

(b)

Figure 11. Relative harmonic distortion on current created at $1 \mathrm{kHz}$ on the blocked voice coil for (a) original motor and (b) magnet-only motor. Measurements realized with a non-moving (blocked) voice coil.

- The presence of eddy currents in iron pieces is significant especially at high frequencies. In contrast, $N d F e B$ magnet used in the modified speaker is a poor conductor whose conductivity is much lower than the conductivity of iron and thus the presence of eddy current is almost eliminated in such a material. Thus, the decrease of eddy currents may explain the large decrease of variations of the blocked parameters with frequency in the case of the magnet-only motor, in comparison with the original motor. Since this frequency variation depends nonlinearly on the position of the voice-coil, it can represent a source of distortion in usual working conditions in case of the original version of the loudspeaker.

- At low frequencies, where the eddy currents are not significant, the original apparent inductance Le of the voice-coil is slightly higher for $x<0$ (coil in) than for $x>0$ (coil out). This is due to the fact that the average permeability of the materials the voice-coil magnetic flux flows through is higher when voice-coil is in iron $(x<0)$ than when it is in air $(x>0)$. In the general linear case, a higher permeability means a higher inductance. In the case of the magnet-only loudspeaker the permeabilities of both the rare-earth magnet and air are almost the same leading to an almost constant permeability, whatever the position of the voice-coil.

The variation of apparent resistance $R e$ with current of the original loudspeaker might be also explained by the presence of eddy currents. One has to remember that eddy currents are often related to a simple heating effect [33]. By measuring the blocked impedance using the concept of the apparent resistance and the apparent inductance, it is shown that the apparent resistance Re (Fig. 7a) increases with the level of the current in the case of the original loudspeaker while it remains almost constant if the motor is replaced by the magnet-only one. This difference can be explained by the heating effect of the eddy currents. The increasing apparent resistance contributes to the increase of the impedance modulus as well as to the reduction of the loudspeaker efficiency through power compression phenomena [37]. Fig. 7b shows that all of these effects can be highly suppressed by using magnet-only motors in loudspeakers.

Moreover, the eddy currents themselves can behave nonlinearly (violating the superposition principle) [38]. They can thus contribute to the nonlinear distortion caused by the traditional loudspeaker motor, in which a soft ferromagnetic material is used. This hypothesis is supported by the fact that the variations of the resistance and the inductance with the current intensity are higher at high frequencies (Figs. 9 and 10), where eddy currents are more significant. Another contribution to the variations of the apparent inductance Le with current of the original 
loudspeaker may be caused by the fact that BH-curve of iron is nonlinear (see Section 2 and [6]). However, the variation of the $\mathrm{BH}$-curve alone cannot explain such a variation of the apparent resistance Re with current and frequency (Fig. 9). The variation of both apparent resistance Re and apparent inductance Le with current can be thus interpreted as a combined effect of the nonlinear BH-curve of the iron in the original loudspeaker motor with the generation of eddy currents in the conductive iron pieces despite the use of shorting rings. However, more scientific evidence would be needed to separate the relative importance of both effects. In the case of the magnetonly loudspeaker, in which the BH-curve is almost linear and in which the eddy currents are highly suppressed, the variation of both, apparent resistance $R e$ and apparent inductance Le with current and frequency are almost eliminated.

In the last experiment, the voice-coil has been kept blocked in its rest position and both original and modified loudspeakers have been supplied with increasing voltage at $1 \mathrm{kHz}$. Even if the voice-coil has been blocked, Fig. 11a shows that the current running through the voice-coil in the case of the original loudspeaker is highly distorted. In the case of the magnet-only loudspeaker Fig. 11b, the distortion of current passing through the blocked voice-coil is highly reduced. This statement confirms the association between the decrease of variation of blocked parameters with a reduction of the current distortion.

\section{CONCLUSION}

In this report, we have presented the results of a comparative study in which a motor of classical electrodynamic loudspeaker has been replaced by a so-called magnet-only motor. Thus, the moving part, including the voicecoil, the diaphragm and the suspensions, has been kept identical during the measurement of both versions of the loudspeaker. Such an experiment has allowed a fair comparison of both motors.

It has been demonstrated that replacing a traditional loudspeaker motor by a magnet-only one, the current distortion of the loudspeaker can be significantly decreased. It has been also shown that the variations of apparent resistance and apparent inductance of the voice-coil with the level of current running through and with displacement are themselves dependent on the frequency. These coupled dependencies and their measurement have been shown to be a good indicator of the nonlinearities of a loudspeaker motor.

The measurements on the original loudspeaker have shown a significant variation of apparent resistance and inductance of the voice-coil with current, displacement and frequency. On the other hand, the measurements on the magnet-only loudspeaker have shown almost no variation whatever the frequency, the current level or the position of the voice-coil in the magnetic circuit.

In addition, by producing a uniform magnetic induction along the entire displacement of the voice-coil, magnetonly structures not only lead to almost constant blocked parameters, but can also assure the force factor to be uniform. By keeping all motor parameters constant, ideal functioning of loudspeakers as described by the simplest linear models can be reached. The case study presented in this paper has shown that the use of magnet-only magnetic circuits in loudspeaker motors can be an important step in loudspeaker linearization. 


\section{References}

[1] H. F. Olson, Elements of acoustical engineering. D. Van Nostrand Company, 1947.

[2] L. L. Beranek, "Acoustics (1954)," New York: Acoust. Soc. Am, 1933.

[3] N. Thiele, "Loudspeakers in vented boxes," in I.R.E. Radio and Electronic Engineering Convention, Proceedings of the IRE Australia, March 1961.

[4] N. Thiele, "Loudspeakers in vented boxes: Part 1," Journal of the Audio Engineering Society, vol. 19, no. 5, pp. 382-392, 1971.

[5] R. H. Small, "Vented-box loudspeaker systems-part 2: Large-signal analysis," Journal of the Audio Engineering Society, vol. 21, no. 6, pp. 438-444, 1973.

[6] W. Klippel, "Tutorial: Loudspeaker nonlinearities - causes, parameters, symptoms," J. Audio Eng. Soc, vol. 54, no. 10, pp. 907-939, 2006.

[7] P. Walker, "Electrostatic loudspeaker with constant current drive," Patent US 3773984 A, 1973.

[8] P. G. Mills and M. J. Hawksford, "Distortion reduction in moving-coil loudspeaker systems using current-drive technology," Journal of the Audio Engineering Society, vol. 37, no. 3, pp. 129-148, 1989.

[9] R. Lian, "Loud speakers," US Patent 3,935,399, 1976.

[10] R. Lian, "Distortion mechanisms in the electrodynamic motor system," in Audio Engineering Society Convention 84, Audio Engineering Society, 1988.

[11] H. Tsuchiya, M. Okada, and T. Saeki, "Reducing harmonic distortion in loudspeakers," in Audio Engineering Society Convention 63, Audio Engineering Society, 1979.

[12] V. G. Carl, "Low distortion dynamic loudspeaker," US patent no 5,151,943, 1992.

[13] H. Mody, "Shorted turn for moving coil motors," US Patent 5,420,468, 1994.

[14] R. Grodinsky, "Distortion reduction in loudspeakers," US Patent 5,357,587, 1994.

[15] L. Goller, "Loudspeaker with short circuit rings at the voice coil," US Patent 5,815,587, 1994.

[16] J. R. Bowler, N. Harfield, N. P. Merricks, and W. J. Woodman, "A theoretical analysis of eddy-current effects in loudspeaker motors," Journal of the Audio Engineering Society, vol. 48, no. 7/8, pp. 668-678, 2000.

[17] W. House, "Transducer motor assembly," US Patent 5,142,260, 1992.

[18] S. Geinseberger and G. Krump, "Loudspeaker having radially magnetized ring," US Patent 6,359,997, 2002.

[19] Y. Ohashi, "Magnetic circuit and speaker," Patent EP 1,553,802 A2, 2005.

[20] G. Lemarquand, B. Richoux, and V. Lemarquand, "Transducteur électrodynamique, applications aux hautparleurs et géophones," PCT/FR2006/051133, 2005.

[21] G. Lemarquand, "New structure of loudspeaker," in Audio Engineering Society Convention 120, Audio Engineering Society, 2006. 
[22] G. Lemarquand, "Ironless loudspeakers," Magnetics, IEEE Transactions on, vol. 43, no. 8, pp. 3371-3374, 2007.

[23] B. Merit, G. Lemarquand, and B. Nemoff, "An ironless low frequency subwoofer functioning under its resonance frequency," in Audio Engineering Society Convention 125, Audio Engineering Society, 2008.

[24] M. Remy, G. Lemarquand, D. Ceruti, G. Guyader, R. Toppi, and M.-F. Six, "Ironless motor loudspeaker: Quantization of the subjective enhanced sound quality," in Audio Engineering Society Convention 129, Audio Engineering Society, 2010.

[25] B. Merit, G. Lemarquand, and V. Lemarquand, "In pursuit of increasingly linear loudspeaker motors," Magnetics, IEEE Transactions on, vol. 45, no. 6, pp. 2867-2870, 2009.

[26] G. Lemarquand and B. Merit, "Magnetic structure for an ironless electrodynamic loudspeaker motor, motors and loudspeakers," Patent US 2010/0172534 A1, 2008.

[27] B. Merit, Contribution to the Identification of the Electrodynamic Loudspeaker Motors Nonlinearities - On the Realization of Magnet-Only Motors (in french). PhD thesis, LAUM, University of Maine, France, 2010.

[28] H. A. Wheeler, "Simple inductance formulas for radio coils," Radio Engineers, Proceedings of the Institute of, vol. 16, no. 10, pp. 1398-1400, 1928.

[29] H. A. Wheeler, "Inductance formulas for circular and square coils," Proceedings of the IEEE, vol. 70, no. 12, pp. 1449-1450, 1982.

[30] W. J. Cunningham, "Non-linear distortion in dynamic loudspeakers due to magnetic effects," The Journal of the Acoustical Society of America, vol. 21, no. 3, pp. 202-207, 1949.

[31] K. Scott, "Variation of the inductance of coils due to the magnetic shielding effect of eddy currents in the cores," Radio Engineers, Proceedings of the Institute of, vol. 18, no. 10, pp. 1750-1764, 1930.

[32] B. Elieli, "The application of an inductively coupled shorted turn and the dual-coil loudspeaker system," in Audio Engineering Society Convention 83, Audio Engineering Society, 1987.

[33] J. Vanderkooy, "A model of loudspeaker driver impedance incorporating eddy currents in the pole structure," Journal of the Audio Engineering Society, vol. 37, no. 3, pp. 119-128, 1989.

[34] W. Klippel, "Scanning the magnetic field of electro-dynamic transducers," in Audio Engineering Society Convention 130, Audio Engineering Society, 2011.

[35] A. Farina, "Simultaneous measurement of impulse response and distortion with a swept-sine technique," in Audio Engineering Society Convention 108, Audio Engineering Society, 2000.

[36] A. Novak, L. Simon, F. Kadlec, and P. Lotton, "Nonlinear system identification using exponential swept-sine signal," Instrumentation and Measurement, IEEE Transactions on, vol. 59, no. 8, pp. 2220-2229, 2010.

[37] D. J. Button, "Heat dissipation and power compression in loudspeakers," Journal of the Audio Engineering Society, vol. 40, no. 1/2, pp. 32-41, 1992.

[38] K. Ishibashi, "Nonlinear eddy current analysis by the integral equation method," Magnetics, IEEE Transactions on, vol. 30, no. 5, pp. 3020-3023, 1994. 